\title{
The Hubbard transition and unsaturated hydrocarbons
}

\author{
Stephen Lee \\ The University of Michigan, Chemistry Department, Ann Arbor, Michigan 48109-1055
}

(Received 27 May 1988; accepted 12 October 1988)

\begin{abstract}
We contrast a simple molecular orbital theory (Hückel) with a simple valence bond theory
(Heisenberg-Dirac). We find for alternant systems in which both models have nondegenerate ground states that both models have ground states belonging to the most symmetrical irreducible representation of the molecular point group. We also find there exist nonalternant systems which have ground states with different irreducible representations. In these latter systems neither the Hückel nor the Heisenberg-Dirac model is sufficient to give a qualitative picture of the molecule. Instead a combined Hückel-Heisenberg-Dirac model (the Hubbard model) must be used. Finally we list some organic unsaturated hydrocarbons, whose ground state changes from one irreducible representation to another as the Hubbard parameters vary.
\end{abstract}

\section{INTRODUCTION}

Although chemists now have at their disposal a wide range of sophisticated techniques ${ }^{1}$ for the calculation of electronic structure, the qualitative models used in understanding this structure tend to be of the most naive sort. Indeed it may be fairly said that among the various qualitative models, only two can be directly described in a quantum mechanical form. They are the one electron molecular orbital theory ${ }^{2}$ and the Heisenberg-Dirac (HD) spin Hamiltonian. ${ }^{3}$

It is commonly assumed that each of these two have their own range of legitimacy. One-electron theories are thought to be applicable to unsaturated hydrocarbon systems, to elemental structures in general, and especially to metals (in the form of band calculations). HD Hamiltonians on the other hand are applied to simple magnetic entities such as $\mathrm{Cr}^{3+}, \mathrm{Fe}^{3+}$, and $\mathrm{Ni}^{2+}$ all of which are embedded in an oxide or halide lattice. The former theory is supposedly valid when bands are wide, i.e., when one-electron terms dominate, the latter when bands are narrow and electron correlation dominates.

It is becoming increasingly apparent however that each of these models can help contribute to the understanding of chemical systems which might have been thought to be the particular province of the other.

For instance, the greatest triumph of the one electron model has been its success in understanding the structure (aromaticity ${ }^{4}$ and bond lengths ${ }^{5}$ ) and reactivity (the Woodward-Hoffmann rules ${ }^{6}$ ) of the unsaturated hydrocarbons. Nevertheless it has been found that the HD model can be useful in explaining some of the properties of these same systems. Thus the HD model correctly predicts that 1 (cyclobutadiene) and 3 are singlets, 2 (trimethylenemethane) and 4 are triplets, and that 5 is a quintet. ${ }^{7}$

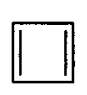

1

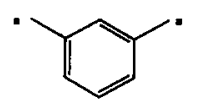

4<smiles>C=C(C)C</smiles>

2

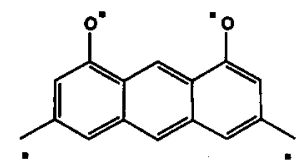

5

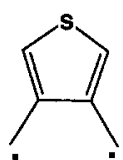

3
(2)

J. Chem. Phys. 90 (5), 1 March 1989 electron and spin models are capable of treating the same chemical systems. ${ }^{8}$ Using models such as the Hubbard model or the PPP models (i.e., models which include both the one-electron and spin models as asymptotic cases), it has been shown for a variety of systems that the Hückel $\left(\Psi_{\text {Hückel }}\right)$ and $\mathrm{HD}\left(\Psi_{\mathrm{HD}}\right)$ ground states for all their intrinsic differences nevertheless bear certain features in common. It has been shown that for the infinite one-dimensional chain no phase transition occurs as one leaves the Hückel asymptotic limit and approaches the HD asymptotic limit. ${ }^{8(a)}$ The same has been found in numerical studies on the two-dimensional square lattice. ${ }^{8(b)}$ Similarly studies on finite systems have also shown that predictions as to spin multiplicity and spin ordering are also independent of the model type. ${ }^{8(\mathrm{c})-8(\mathrm{e})}$ In this paper we establish several results; which further corroborate the interconnection which exists between Hückel and HD theory. We show that when an alternant chemical system does not have a degenerate Hückel ground state then both $\Psi_{\text {Hückel }}$ and $\Psi_{\text {HD }}$ belong to the same irreducible representation (ir) of the symmetry group of the molecule. We also show that for nonalternant systems, there exists molecules for which this is not true, i.e., $\Psi_{\mathrm{HD}}$ and $\Psi_{\text {Hückel }}$ are irreconcilably different. These exceptional systems are the particular concern of the current work. It is found that they are systems whose chemical behavior cannot be understood by either a one-electron theory (such as the Hückel model) nor a valence bond theory (such as the HD model). Instead a combined valence bond-molecular orbital theory must be used.

\section{DEFINITIONS}

The Hückel Hamiltonian is a one-electron Hamiltonian. It may be considered to be acting on a space of atomic orbitals. In this case

$$
H_{\text {Hückel }}=\sum_{i, j}\left|x_{i}\right\rangle \beta_{i j}<x_{j} \mid,
$$

where $\left|x_{i}\right\rangle$ and $\left|x_{j}\right\rangle$ are atomic orbitals and

$$
\beta_{i j}= \begin{cases}\beta & \text { if } x_{i} \text { and } x_{j} \text { are nearest neighbors } \\ 0 & \text { otherwise }\end{cases}
$$

Alternatively, the Hückel Hamiltonian may be considered to be acting on $n$-electron wave functions (we shall call such 
$n$-electron wave functions SDs for Slater determinants). In this case

$$
H_{\mathrm{Hückel}}=\sum_{i, j} \beta_{i j} a_{i \alpha}^{+} a_{j \alpha},
$$

where $a_{i \alpha}^{+}$and $a_{i \alpha}$ are, respectively, the creation and annihilation operators for $x_{i \alpha}$ and where the $\alpha$ index denotes whether the $i$ orbital is up-spin $(\alpha=+)$ or down-spin $(\alpha=-)$.

The Heisenberg-Dirac spin Hamiltonian (HD) acts only on a space of SDs

$$
H_{\mathrm{HD}}=-\sum_{i, j} J_{i j} \mathbf{S}_{i} \cdot \mathbf{S}_{j},
$$

where $\mathbf{S}_{i}$ and $\mathbf{S}_{j}$ are spin $1 / 2$ operators indexed by atomic orbital and

$$
J_{i j}=\left\{\begin{array}{ll}
J & \text { if } \beta_{i j}=\beta \\
0 & \text { otherwise }
\end{array} .\right.
$$

Both Hückel and HD Hamiltonians are asymptotic cases of the Hubbard Hamiltonian':

$$
H_{\mathrm{Hubbard}}=\sum_{i>j} \beta_{i j} a_{i \alpha}^{+} a_{j \alpha}+U \sum_{i} a_{i+}^{+} a_{i-}^{+} a_{i-} a_{i+} .
$$

Thus when $U=0$,

$$
H_{\text {Hubbard }}=H_{\text {Hücke }}
$$

and when $U \gg|\beta|$,

$$
P H_{\text {Hubbard }} P=H_{\mathrm{HD}} \text {, }
$$

where $P$ is the projection operator onto the space where every SD has single occupation of every spatial atomic orbital. The following two geometrical concepts are also important:

(1) Alternant. ${ }^{10} \mathrm{~A}$ Hamiltonian is alternant with respect to a given basis set when the basis vectors may be divided into two sets, the starred set and the unstarred set, in such a way that all nonzero off-diagonal terms connect starred basis vectors to unstarred basis vectors.

(2) Connected. A Hamiltonian is connected with respect to a given basis set when the basis set does not create a block-diagonal Hamiltonian.

Finally in Table I we state the notational conventions which will be used in this paper.

\section{ALTERNANT SYSTEMS}

As we have discussed in an earlier paper ${ }^{8(e)}$ the localized portion of $\Psi_{\text {Hückel }}\left(P \Psi_{\text {Hückel }}\right)$ and $\Psi_{\mathrm{HD}}$ bear a strong resemblance to one another. In Table II we give an illustration of this for 6 octatetraene, $\mathrm{C}_{8} \mathrm{H}_{10}$.

$$
\text { רon }
$$

This similarity between $P \Psi_{\text {Hückel }}$ and $\Psi_{\mathrm{HD}}$ indicates that the change in $\Psi_{\text {Hubbard }}$ as the $U / \beta$ ratio is altered is a gradual one. One further indication of this is found in the following

\begin{tabular}{|c|c|}
\hline$x_{i}, \phi_{i}, i$ & Refer to atomic orbitals ${ }^{\mathrm{a}}$ \\
\hline$i, j, k$ & Indices of atomic orbitals \\
\hline$g, f$ & $\begin{array}{l}\text { Group elements of the groups } \\
G \text { and } F \text {. Point groups element } \\
\text { can be thought to act either } \\
\text { on atoms or SDs. }\end{array}$ \\
\hline (12) & $\begin{array}{l}\text { Permutation of atom (atomic } \\
\text { orbital) } 1 \text { and } 2\end{array}$ \\
\hline$a^{+}, a$ & $\begin{array}{l}\text { Creation and annihilation } \\
\text { operators }\end{array}$ \\
\hline$\alpha$ & $\begin{array}{l}\text { Spin index, } \alpha=+ \text { for up-spin } \\
\alpha=- \text { for down-spin }\end{array}$ \\
\hline \multirow[t]{2}{*}{$|1 \overline{2}|$} & $\begin{array}{l}\text { Refers to the Slater } \\
\text { determinant }\end{array}$ \\
\hline & $\left|\begin{array}{ll}x_{1+}\left(r_{1}\right) & x_{2-}\left(r_{1}\right) \\
x_{1+}\left(r_{2}\right) & x_{2-}\left(r_{2}\right)\end{array}\right|$ \\
\hline$P$ & $\begin{array}{l}\text { Projection operator onto } \\
\text { localized space, e.g., } \\
P\{|1 \overline{1}|+|1 \overline{2}|\}=|1 \overline{2}|\end{array}$ \\
\hline$\psi$ & Refers to a Slater determinant \\
\hline LSD & $\begin{array}{l}\text { Localized Slater determinant } \\
\text { if } \psi_{y} \text { is an LSD then } P \psi_{y}=\psi_{y}\end{array}$ \\
\hline$\Phi$ & A molecular orbital \\
\hline$t$ & An arbitrary vector \\
\hline$\Psi_{\text {Hückel }}, \Psi_{\mathrm{HD}}, \Psi_{\text {Hubbard }}$ & $\begin{array}{l}\text { The ground state of the } \\
\text { Hückel, HD, and Hubbard } \\
\text { Hamiltonians }\end{array}$ \\
\hline
\end{tabular}
result.

Theorem: Consider a connected alternate system with an even number of atoms. Let $\Psi_{\text {Hückel }}$ be nondegenerate. Let the system be symmetric with respect to a point group $G$, where $G$ is a three-dimensional point group. Under these
TABLE I. Key to symbols.

${ }^{a}$ Where no ambiguity exists the $x$ or $\phi$ symbol is dropped. An example is $|\overline{1}|=\left|x_{1+} x_{2-}\right|$

conditions both $\Psi_{\text {Hïckel }}$ and $\Psi_{\text {HD }}$ belong to the most symmetrical representation of $G$. The proof of this result is given in the Appendix I.

The Hubbard correlation diagram for alternant nondegenerate systems (such as octatetraene) is therefore of the form shown in Fig. 1 (a). The true chemical system lies intermediate between the Hückel and HD antipodes. As the alteration in $\Psi_{\text {Hubbard }}$ is gradual (i.e., analytic), it is reasonable that both the Hückel model and the HD model would be suitable starting points for calculations. Both initial calculations could then be improved upon by perturbation techniques or another similar method. By contrast if one were to study a system whose Hubbard correlation diagram is of the form given in Fig. 1(b) much greater care would have to be taken in the initial method of calculation. In those systems where $|\beta| \gg U$, Hückel theory would have to be used while if $U \gg|\beta|$, HD theory would be required. This latter situation would certainly be the case for narrow $d$-band transition metal insulators. The situation is even more complex for unsaturated hydrocarbons. Recent effective Hamiltonian 
TABLE II. Comparison of $\Psi_{\text {Hückel }}$ and $\Psi_{\mathrm{HD}}$ for octatetraene.

\begin{tabular}{|c|c|c|}
\hline $\begin{array}{l}\text { Down-spin } \\
\text { atoms }^{\mathrm{a}}\end{array}$ & $\Psi_{\text {Hüekel }}{ }^{\mathrm{b}}$ & $\Psi_{\mathrm{HD}}$ \\
\hline $5,6,7,8$ & 0.001 & 0.000 \\
\hline $4,6,7,8$ & -0.001 & -0.004 \\
\hline $4,5,7,8$ & 0.000 & 0.016 \\
\hline $4,5,6,8$ & -0.013 & -0.026 \\
\hline $4,5,6,7$ & 0.013 & 0.014 \\
\hline $3,6,7,8$ & 0.000 & 0.016 \\
\hline $3,5,7,8$ & -0.030 & -0.084 \\
\hline $3,5,6,8$ & 0.127 & 0.157 \\
\hline $3,5,6,7$ & -0.098 & -0.089 \\
\hline $3,4,7,8$ & 0.030 & 0.094 \\
\hline $3,4,6,8$ & -0.185 & -0.300 \\
\hline $3,4,6,7$ & 0.156 & 0.194 \\
\hline $3,4,5,8$ & 0.070 & 0.131 \\
\hline $3,4,5,7$ & -0.070 & -0.158 \\
\hline $3,4,5,6$ & 0.000 & 0.038 \\
\hline $2,6,7,8$ & -0.013 & -0.026 \\
\hline $2,5,7,8$ & 0.127 & 0.157 \\
\hline $2,5,6,8$ & -0.351 & -0.323 \\
\hline $2,5,6,7$ & 0.236 & 0.192 \\
\hline $2,4,7,8$ & -0.185 & -0.300 \\
\hline $2,4,6,8^{c}$ & 1.000 & 1.000 \\
\hline $2,4,6,7$ & -0.802 & -0.670 \\
\hline $2,4,5,8$ & -0.566 & -0.493 \\
\hline $2,4,5,7$ & 0.623 & 0.622 \\
\hline $2,4,5,6$ & -0.070 & -0.158 \\
\hline $2,3,7,8$ & 0.070 & 0.131 \\
\hline $2,3,6,8$ & -0.566 & -0.493 \\
\hline $2,3,6,7$ & 0.508 & 0.372 \\
\hline $2,3,5,8$ & 0.634 & 0.465 \\
\hline $2,3,5,7$ & -0.802 & -0.670 \\
\hline $2,3,5,6$ & 0.156 & 0.195 \\
\hline $2,3,4,8$ & -0.151 & -0.117 \\
\hline $2,3,4,7$ & 0.236 & 0.192 \\
\hline $2,3,4,6$ & -0.098 & -0.089 \\
\hline $2,3,4,5$ & 0.013 & 0.014 \\
\hline
\end{tabular}

"We list in this column only the down-spin electrons. Thus the first row

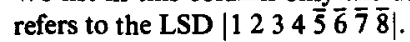

${ }^{b}$ Both $\Psi_{\text {Huskel }}$ and $\Psi_{\mathrm{HD}}$ are singlets.

' The largest coefficient is set equal to one.

a.

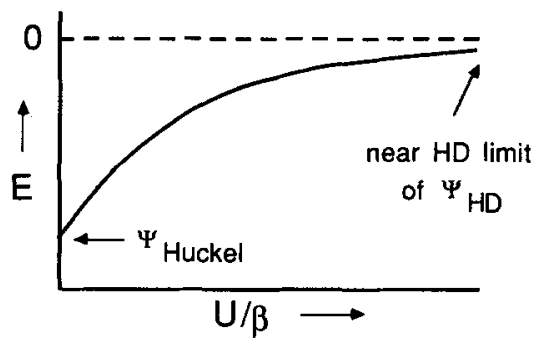

b.

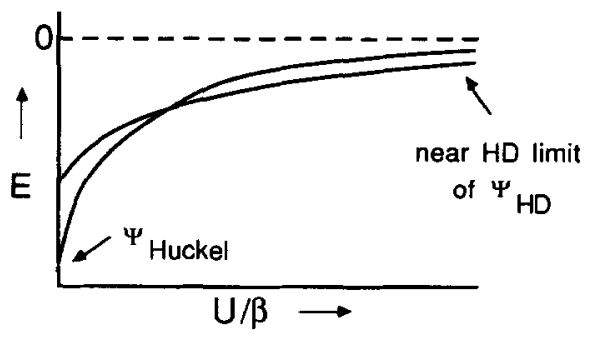

FIG. 1. (a) The Hubbard ground state energy as a function of $U / \beta$ for a system without a Hubbard transition. (b) The Hubbard ground state energy for a system with a Hubbard transition. work " shows that a reasonable $|U / \beta|$ ratio is between 1 and 4. It therefore appears that neither Hückel theory nor HD theory by themselves would be sufficient.

\section{HUBBARD TRANSITIONS}

In the current work we are interested in investigating systems which have Hubbard correlation diagrams like that shown in Fig. 1(b). We are therefore interested in finding systems where $\Psi_{\text {Hückel }}$ and $\Psi_{\mathrm{HD}}$ belong to different irreducible representations. One of the simplest such systems is found in the $\pi$ orbitals of 7 , pentalene $\left(\mathrm{C}_{8} \mathrm{H}_{6}\right){ }^{12}$<smiles>C1=CC2=CC=CC2=C1</smiles>

In Table III we contrast the localized portion of $\Psi_{\text {Hückel }}$ and $\Psi_{\mathrm{HD}}$. Unlike for the alternant hydrocarbons such as 6 , $\Psi_{\text {Hückel }}$ and $\Psi_{\text {HD }}$ bear no resemblance. Inspection shows that the $\Psi_{\text {Hückel }}$ of pentalene is of $A_{1 g}$ symmetry while $\Psi_{\mathrm{HD}}$ is of the $B_{1 g}$ symmetry. We show in Fig. 2 the Hubbard correlation diagram for $\Psi_{\text {Hückel }}$ and $\Psi_{\mathrm{HD}}$. It may be seen that pentalene indeed undergoes a transition in its ground state for a certain critical value of $U / \beta$. We term this transition a Hubbard transition.

The geometrical factors responsible for the transition are straightforward once one makes the assumption that the lowest energy localized SD (LELSD) does not have a zero coefficient in $\Psi_{\mathrm{HD}}$. Thus, for example, in pentalene if we label the atoms as indicated in 8 there are two LELSDs.<smiles>C1=CC2=CC=CC2=C1</smiles>

8

The first has up-spin electrons on atoms $1,3,5$, and 7 and down-spin electrons on atoms 2,4,6, and 8. The second LELSD has down-spin electrons on atoms 1,3,5, and 7 and up-spin electrons on 2,4,6, and 8. These are shown in Fig. 3 along with the LELSDs of benzene and $9, \mathrm{a} \mathrm{C}_{14} \mathrm{H}_{12}$ singlet biradical isomer.<smiles>Cc1cccc(-c2ccccc2)c1</smiles>

Following the conventions listed in Table $I$ the former SD is $|1 \overline{2} 3 \overline{4} 5 \overline{6} 7 \overline{8}|$ while the latter is $|\overline{1} 2 \overline{3} 4 \overline{5} 6 \overline{7} 8|$.

Under point group symmetry operations LELSDs are always mapped into LELSDs. Pentalene has among its symmetry operations $\sigma_{x}$, a mirror plane normal to the $x$ axis, and $i$, an inversion center. The mirror plane $\sigma_{x}$ corresponds to the permutation (17) (26) (35) while $i$ corresponds to (48) (37) (26) (15). Therefore,

$$
\sigma_{x}|1 \overline{2} 3 \overline{4} 5 \overline{6} 7 \overline{8}|=-|1 \overline{2} 3 \overline{4} 5 \overline{6} 7 \overline{8}|
$$

and

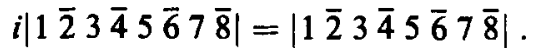


TABLE III. Comparison of $\Psi_{\text {Hǘckel }}, \Psi_{\mathrm{HD}}$, and first excited states of $H_{\text {Hückel }}$ and $H_{\mathrm{HD}}$ for pentalene.

\begin{tabular}{|c|c|c|c|c|}
\hline $\begin{array}{l}\text { Down-spin } \\
\text { atoms }^{\mathrm{a}}\end{array}$ & $\Psi_{\text {Hückel }}$ & $\begin{array}{l}\text { First excited } \\
\text { state of } H_{\mathrm{HD}}\end{array}$ & $\Psi_{\mathrm{HD}}$ & $\begin{array}{l}\text { First excited } \\
\text { state of } H_{\text {Hückel }}\end{array}$ \\
\hline $5,6,7,8$ & 0.008 & 0.024 & 0.021 & 0.007 \\
\hline $4,6,7,8$ & -0.038 & -0.082 & -0.098 & -0.053 \\
\hline $4,5,7,8$ & 0.000 & 0.000 & 0.0157 & 0.101 \\
\hline $4,5,6,8$ & 0.038 & 0.082 & -0.098 & -0.053 \\
\hline $4,5,6,7$ & -0.008 & -0.024 & 0.021 & 0.007 \\
\hline $3,6,7,8$ & -0.162 & -0.106 & 0.130 & 0.125 \\
\hline $3,5,7,8$ & 0.698 & 0.638 & -0.497 & -0.425 \\
\hline $3,5,6,8$ & -0.581 & -0.642 & 0.447 & 0.350 \\
\hline $3,5,6,7$ & 0.038 & 0.082 & -0.098 & -0.057 \\
\hline $3,4,7,8$ & -0.404 & -0.304 & 0.237 & 0.249 \\
\hline $3,4,6,8$ & 0.442 & 0.389 & -0.402 & -0.444 \\
\hline $3,4,6,7$ & 0.162 & 0.106 & 0.130 & 0.125 \\
\hline $3,4,5,8$ & 0.000 & 0.000 & 0.106 & 0.149 \\
\hline $3,4,5,7$ & -0.294 & -0.266 & -0.003 & -0.074 \\
\hline $3,4,5,6$ & 0.106 & 0.096 & -0.051 & -0.000 \\
\hline $2,6,7,8$ & 0.294 & 0.334 & -0.003 & -0.074 \\
\hline $2,5,7,8$ & -1.000 & -1.000 & 0.322 & 0.392 \\
\hline $2,5,6,8$ & 0.698 & 0.638 & -0.497 & -0.425 \\
\hline $2,5,6,7$ & 0.000 & 0.000 & 0.160 & 0.101 \\
\hline $2,4,7,8$ & 0.442 & 0.389 & -0.402 & -0.444 \\
\hline $2,4,6,8^{\mathrm{c}}$ & 0.000 & 0.000 & 1.000 & 1.000 \\
\hline $2,4,6,7$ & -0.698 & -0.638 & -0.497 & -0.425 \\
\hline $2,4,5,8$ & -0.442 & -0.389 & -0.402 & -0.444 \\
\hline $2,4,5,7^{c}$ & 1.000 & 1.000 & 0.322 & 0.392 \\
\hline $2,4,5,6$ & -0.294 & -0.334 & -0.003 & -0.007 \\
\hline $2,3,7,8$ & 0.162 & 0.106 & 0.130 & 0.125 \\
\hline $2,3,6,8$ & -0.698 & -0.642 & -0.497 & -0.425 \\
\hline $2,3,6,7$ & 0.404 & 0.304 & 0.236 & 0.250 \\
\hline $2,3,5,8$ & 0.581 & 0.642 & 0.447 & 0.350 \\
\hline $2,3,5,7$ & -0.442 & -0.389 & -0.402 & -0.444 \\
\hline $2,3,5,6$ & 0.000 & 0.000 & 0.106 & 0.149 \\
\hline $2,3,4,8$ & -0.038 & -0.082 & -0.098 & -0.057 \\
\hline $2,3,4,7$ & -0.162 & -0.106 & 0.130 & 0.125 \\
\hline $2,3,4,6$ & 0.294 & 0.331 & 0.003 & -0.074 \\
\hline $2,3,4,5$ & -0.106 & -0.171 & -0.051 & -0.000 \\
\hline
\end{tabular}

${ }^{a}$ We list in this column only the down-spin electrons. Thus the first row refers to the LSD $|12345 \overline{5} \overline{6} \overline{7}|$.

${ }^{\mathrm{D}} \boldsymbol{\Psi}_{\text {Hückel }}, \Psi_{\mathrm{HD}}$, and both first excited states are singlets.

${ }^{\mathrm{c}}$ The largest coefficient in each column is set equal to one.

It is of interest that $\sigma_{x}$ transforms the LELSD in Eq. (9) into the negative of itself. Therefore if one is to have a nonzero amount of the LELSD in the ground state of the system, the ground state cannot belong to the most symmetrical irreducible representation. But $\Psi_{\text {Hückel }}$ of pentalene does belong to the most symmetrical irreducible representation. Therefore a Hubbard transition must occur.
Huckel
Limit
HD
Limit

10.0

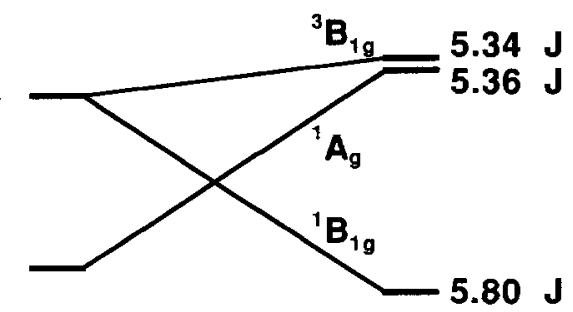

FIG. 2. Correlation diagram between the Hückel and HD Hamiltonians for the $\pi$ system of pentalene. a.<smiles></smiles><smiles>c1ccccc1</smiles>

b.<smiles>c1cc2occc2o1</smiles>

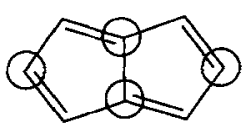

c.<smiles></smiles>

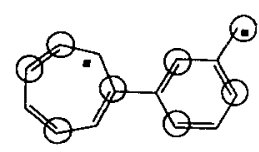<smiles></smiles>

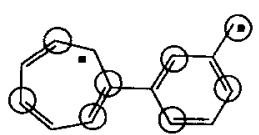

FIG. 3. (a) The LELSDs for benzene. Circled atoms are atoms with an upspin $\pi$ electron. Uncircled atoms are atoms with down-spin $\pi$ electrons. (b) LELSDs of pentalene (c) LELSDs of 9. We show here only the LELSDs which belong to the $S_{z}=1$ manifold. See Fig. 5 for the $S_{z}=0$ manifold LELSDs. 
a.<smiles>c1ccc2ccccc2c1</smiles>

b.<smiles>CC(C)=C1C=C1</smiles>

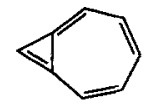

e.

d.
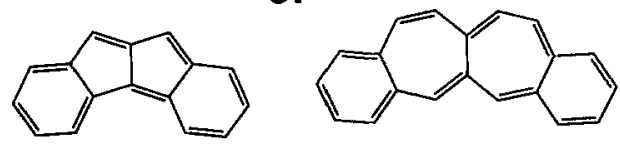

FIG. 4. Systems which have a Hubbard transition.

\section{SYSTEMS WITH HUBBARD TRANSITIONS}

We therefore can anticipate the Hubbard transition in pentalene because in pentalene three conditions are met:

(1) The molecule has a nondegenerate $\Psi_{\text {Hückel }}$.

(2) The molecule has a nontrivial point group.

(3) There exist symmetry elements which permute the

LELSDs into the negative of themselves.

By the same logic the molecules shown in Fig. 4 also would appear to have a Hubbard transition.

Slightly more complicated is the case where the permutation transforms one LELSD into another. One intuitive way of treating such cases is by recognizing that nonalternant systems are often only slightly perturbed alternant systems. Thus pentalene is just the system 10 where one extra $\pi$ bond has been made, while 9 has just one $\pi$ bond more than 11-14. Of these, 12-14 are the alternant systems most closely related to 9 which have a singlet $\Psi_{\text {HD }}$ ground state. ${ }^{13}$<smiles></smiles>

10<smiles>C=C/C=C(\CC=C)c1cccc(C)c1</smiles>

12<smiles>C=C[C]C=C(C=C)c1cccc(C)c1</smiles>

11

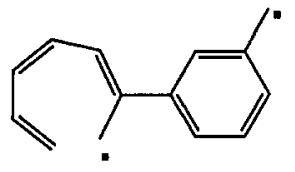

13

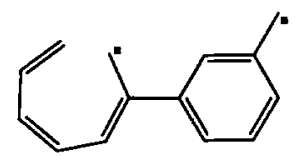

14

If all the alternant systems which are most closely related to the nonalternant system in question have in their respective ground states identical phases for the various LELSDs it is reasonable to assume that in the parent nonalternant system the same phase relations are maintained. As an example we consider 9. In Table IV we list the phases of the SDs of the three LELSDs $\psi_{1}, \psi_{2}$, and $\psi_{3}$ where

$$
\begin{aligned}
& \psi_{1}=|1 \overline{2} 34 \overline{5} 6 \overline{7} 8 \overline{9} 10 \overline{1} 112 \overline{1} 3 \overline{1} 4|,
\end{aligned}
$$

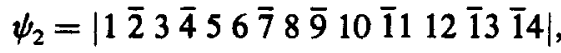

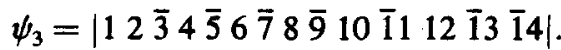

TABLE IV. Phase of $\psi_{1}, \psi_{2}$, and $\psi_{3}$ for $12-14$. $^{\mathrm{a}}$

\begin{tabular}{lccc}
\hline \hline & 12 & 13 & 14 \\
\hline Phase of $\psi_{1}$ in $\Psi_{\mathrm{HD}}$ for & + & + & + \\
Phase of $\psi_{2}$ in $\Psi_{\mathrm{HD}}$ for & - & - & - \\
Phase of $\psi_{3}$ in $\Psi_{\mathrm{HD}}$ for & - & - & - \\
\hline
\end{tabular}

$\psi_{1}, \psi_{2}$, and $\psi_{3}$ are given in Eqs. (11)-(13). The atom labels for these equations are shown in 9.

As we see from Table IV $, \psi_{1}, \psi_{2}$, and $\psi_{3}$ have identical phases for systems 12-14. We therefore expect that for 9 the coefficients of LELSDs in $\Psi_{\mathrm{HD}}$ are

$$
\Psi_{\mathrm{HD}}=a \psi_{1}-b\left(\psi_{2}+\psi_{3}\right)+\text { other LSDs, }
$$

where $\psi_{1}, \psi_{2}$, and $\psi_{3}$ are those given in Fig. 5 and where $a$ and $b$ are positive real numbers.

This is further corroborated by the matrix elements:

$$
\begin{aligned}
& \left\langle\psi_{1}\left|H_{\mathrm{HD}}\right| \psi_{2}\right\rangle=-J, \\
& \left\langle\psi_{1}\left|H_{\mathrm{HD}}\right| \psi_{3}\right\rangle=-J, \\
& \left\langle\psi_{2}\left|H_{\mathrm{HD}}\right| \psi_{3}\right\rangle=0 .
\end{aligned}
$$

As the three LSDs are the lowest energy ones, the above relations imply in first order perturbation theory that the phase relation given in Eq. (14) is the correct one for $\Psi_{\mathrm{HD}}$.

We now note that there is a hidden symmetry element in 9, the permutation (16) (25) (34). Furthermore we note,

$$
\text { (16) (25) (34) } \psi_{1}=-\psi_{1}
$$

while

$$
\text { (16) (25) (34) }\left[\psi_{2}+\psi_{3}\right]=-\left[\psi_{2}+\psi_{3}\right]
$$

As the $\Psi_{\text {Hückel }}$ of 9 is nondegenerate, both relations indicate that 9 also undergoes a Hubbard transition. Direct verification for 9 is difficult. On the other hand we can numerically examine the related system 15 .

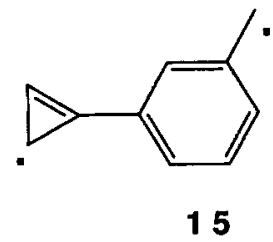

Calculation reveals 15 does indeed undergo a Hubbard transition. ${ }^{13}$

These simple ideas can be reformulated in the following useful way. In certain cases the alternant systems which are most closely related to the nonalternant molecule under in-

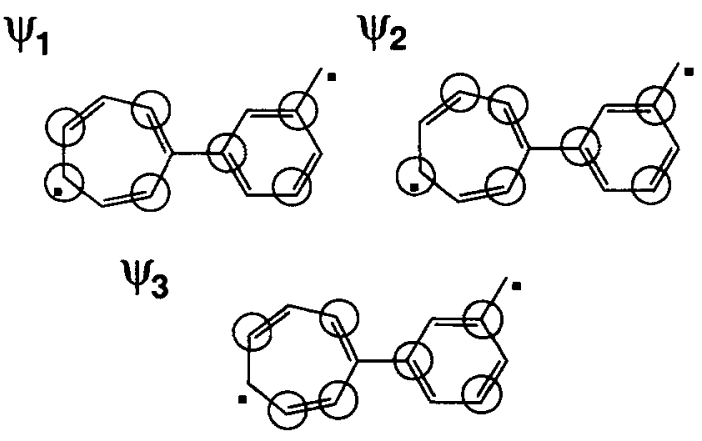

FIG. 5. LELSDs for 9. $\psi_{1}, \psi_{2}$, and $\psi_{3}$ are given in Eqs. (11)-(13). 


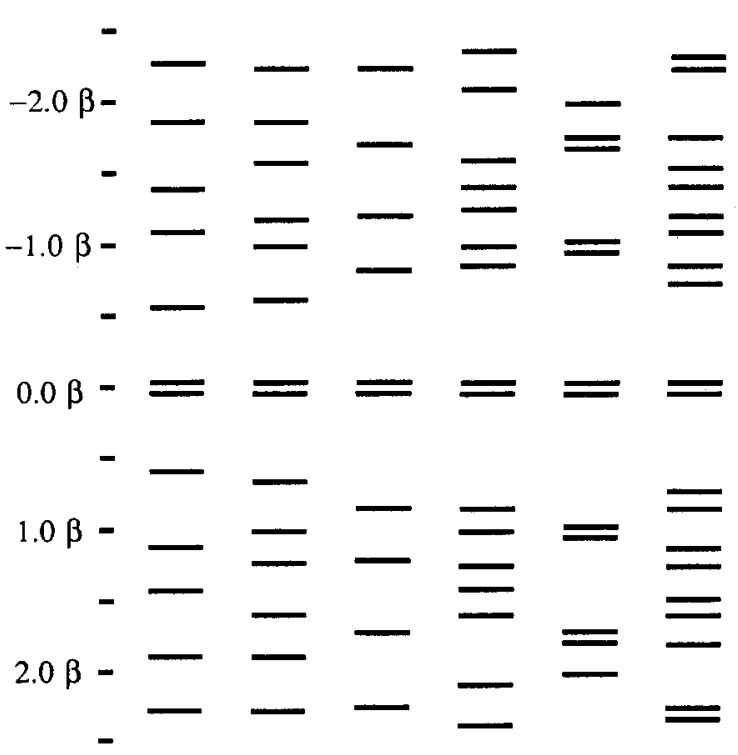

FIG. 6. $\pi$ molecular orbital energies for systems 12, 13, and 16-19. Note in all cases $\Psi_{\text {Huckel }}$ is degenerate, as there exist molecular orbitals which are zero eigenvalued.

vestigation maintain all the symmetry elements of the parent nonalternant systems. Examples of this are seen in $\mathbf{1 0}$ and $\mathbf{1 2}$. If we find in the nonalternant system that $\Psi_{\mathrm{HD}}$ does not belong to the most symmetrical representation of the point group this implies that the same is true for the $\Psi_{\text {HD }}$ of the related alternant systems. From the result of Appendix I we therefore conclude that $\Psi_{\text {Hückel }}$ for the alternant system must have degeneracies. In other words there exists a molecular orbital such that $H_{\text {Hückel }} \Psi=0$. We show in Fig. 6 that this is indeed the case for the systems 12,13, and 16-19.

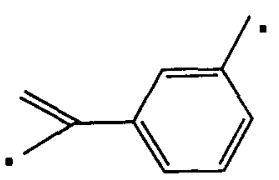

16

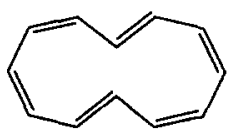

18

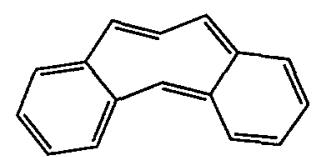

17

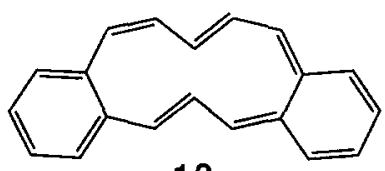

19

\section{BROKEN SYMMETRY}

We now turn to the chemical consequences of the Hubbard transition. The most interesting consequence occurs when the true effective $U / \beta$ ratio is near the $U / \beta$ ratio at which the ground states cross. Under these conditions we should observe symmetry breaking, where the molecule lowers its symmetry so as to be able to mix effectively the two low lying eigenstates. For example, in the case of 7, pentalene, the two low-lying states are of $A_{1 g}$ and $B_{1 g}$ symmetry. Mixing of the two states is only allowed in the symmetry lowering from $D_{2 h}$ to $C_{2 h}$. Pentalene should therefore distort as shown in 20. We show the qualitative energetic effects in Fig. 7.

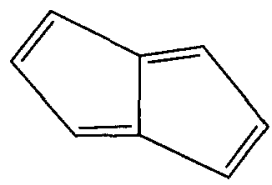

This symmetry distortion relies though on the proximity of the orbital Hubbard $U / \beta$ transition ratio (see Fig. 7) and the true effective $U / \beta$ ratio of the system. As we noted earlier the true effective $U / \beta$ ratios lie between 1 and 4 . The lower limit is in the case of nonorthogonal $p \pi$ orbitals and the upper limit for orthogonal ones. We must therefore contrast the $U / \beta$ value to the $U / \beta$ value at which the $A_{1 g}$ and $B_{1 g}$ states cross. Unfortunately, it is difficult to directly calculate this number for the eight atom pentalene system. In the case of simpler four and six atom systems, though, direct calculation of the $U / \beta$ value at which the Hubbard transition take place is possible. We discuss in Appendix II how the $U / \beta$ value of the Hubbard transition can be estimated from the differences in energy $\Delta E_{\mathrm{HD}}$ and $\Delta E_{\text {Hückel }}$ (see Fig. 7 ), where $\Delta E_{\text {Hückel }}$ is the difference in energy between $\Psi_{\text {Hückel }}$ and the Hückel eigenstate which correlates with $\Psi_{\mathrm{HD}}$, and $\Delta E_{\mathrm{HD}}$ is the difference in energy between $\Psi_{\mathrm{HD}}$ and the HD eigenstate which correlates with $\Psi_{\text {Hückel }}$. As we show in Appendix B a reasonable estimate of the $U / \beta$ value corresponding to the Hubbard transition is

$$
\frac{U}{\beta}=\frac{1}{\Delta E_{\mathrm{HD}}}+2 \cdot \Delta E_{\mathrm{Hückel}}
$$

in the case of pentalene $\Delta E_{\mathrm{HD}}=0.43 \mathrm{~J}$ and $\Delta E_{\mathrm{Hückel}}$ $=0.47 \beta$. Therefore we anticipate the Hubbard transition occurs at around a $U / \beta$ ratio of 2.0 to 4.0. This places us in the middle range of calculated $U / \beta$ ratios. Therefore it appears reasonable that symmetry breaking due to the Hubbard transition should take place.

This predicted symmetry breaking appears to be present in pentalene. While pentalene has only been observed as a fugitive species at $-196^{\circ} \mathrm{C}$, two substituted pentalenes 21 and 22 have known crystal structures. ${ }^{14(a)}$<smiles>CC(C)(C)C1=CC2=C(C(C)(C)C)C=C(C(C)(C)C)C2=C1</smiles>

21<smiles>CC(=O)OC(C)=O</smiles>

22
In Fig. 8 we show the reported bond distances. It should be noted that in the current work we have considered the $\pi$ system as perfectly distinct from the $\sigma$-system. Thus we are not able to study either $\sigma-\pi$ mixing nor deviation from planarity. Nevertheless the experimental evidence indicates that the $C_{2 h}$ planar geometry is of lower energy than the $D_{2 h}$ geometry.

The same symmetry breaking is also observed in heptalene. Again only the crystal structures of the substituted heptalenes 23 and 24 are known. ${ }^{14(b), 14(c)}$ 


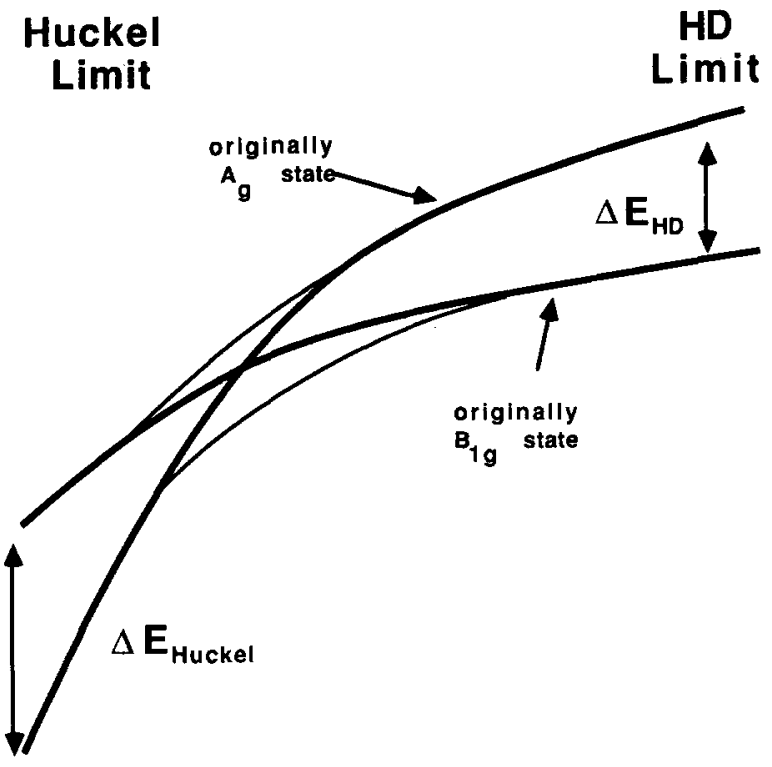

FIG. 7. Effect of distortion from $D_{2 h}$ to $C_{2 h}$ symmetry for pentalene. With dark lines, we show the crossing which occurs between the Hückel and HD limits in the case of $D_{2 n}$ symmetry. With thinner lines, we show the avoided crossing of the $C_{2 h}$ symmetry system.<smiles>CC(=O)OC(C)=O</smiles>

23

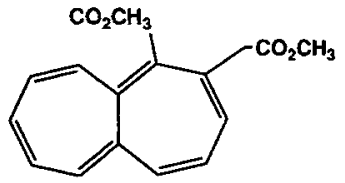

24
Finally it should be noted that the symmetry breaking which we have discussed is very different from the symmetry breakings previously discussed for unsaturated hydrocarbons. There have been two previously discussed forms of symmetry breaking. In the case of cyclobutadiene the $\pi$ system distorts so as to relieve the degeneracy of the Hückel molecular orbitals. This is a Jahn-Teller distortion. a.<smiles>CC(C)(C)C1=C[C@@H]2C(C(C)(C)C)=C[C@H](C(C)(C)C)[C@H]2C=C1</smiles>

c.

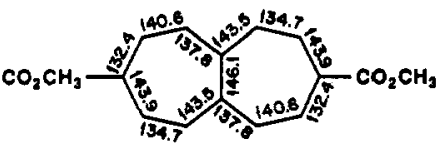

b.

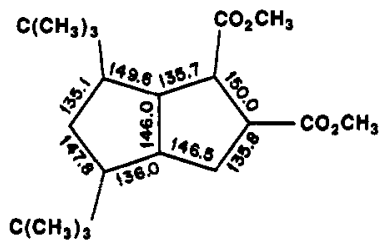

d.

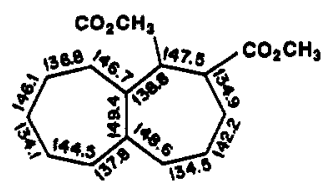

FIG. 8. Reported bond distances for pentalene and heptalene derivatives. Distances are given in p.m. (data is taken from Ref. 14).
A similar distortion occurs in the large $n[n]$-annulenes. ${ }^{15}$ It corresponds to the solid state Peierls distortion. In this case as $n$ increases in size the HOMO and LUMO become sufficiently close in energy that again a pairing distortion by mixing HOMO and LUMO together stabilizes the system. This distortion is seen in the large $[n]$-annulenes where $n>20$ atoms.

Both the Jahn-Teller and the Peierls distortions are effects which can be understood with a one electron picture. This is in contrast with the Hubbard transition where the distortion is due to a degeneracy which is only present in full configuration interaction space. Indeed there is no indication in the Hückel model of either pentalene or heptalene that a degeneracy is present. In fact, early workers in the field were of the opinion that pentalene and heptalene were aromatic systems.

\section{RING PARAMAGNETISM}

One well studied effect in ring systems is the deviation of the magnetic susceptibility when compared to the sum of the atomic susceptibilities. It has been found in $4 n$-systems the deviation necessitates the inclusion of a paramagnetic term. ${ }^{16}$ One particularly elegant explanation of this effect is based on orbital ring angular momentum. The idea is a simple one. In undistorted cyclooctatetraene the HOMO and LUMO are a combination of the two state $\Phi_{a}, 25$ and $\Phi_{b}, 26$.

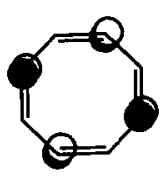

25

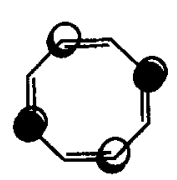

26
It has been pointed out that $\Phi_{a} \pm i \Phi_{b}$ closely resemble the states with $L_{z}= \pm 2$ for systems with cylindrical symmetry. Thus under a magnetic field there will be a paramagnetic contribution from this effect.

This interesting observation has been used to explain not only the paramagnetic deviation in $4 n$-annulenes but in pentalene and heptalene as well. Thus 9, 10-dimethyldibenzopentalene has a paramagnetic deviation of $-14 \times 10^{-6}$ $\mathrm{cm}^{3} / \mathrm{mol}$ and (compared to $-5 \times 10^{-6}$ for [16]-annulene) ${ }^{16}$ In turning to the Hückel diagram for pentalene we see that it differs from [8]-annulene in one important way. The central degeneracy in [8]-annulene has been lifted. We therefore ought to see quenching of orbital angular momentum. If the above elegant explanation for the paramagnetism of $4 n$ systems is correct we ought not see the effect in either pentalene or heptalene. It is interesting though that in the Hubbard model this quenching of ring orbital angular momentum is partially lost. In pentalene we have two low-lying singlet energy states. Mixing of these two states does permit the formation of states which have nonzero expectation values with respect to the operator $\mathscr{L}_{z}$ :

$$
\mathscr{L}_{z}=\left|\psi_{c}\right\rangle\left\langle\psi_{c}|-| \psi_{d}\right\rangle\left\langle\psi_{d}\right|
$$

$\Psi_{c}$ and $\psi_{d}$ are shown in Fig. 9 . 


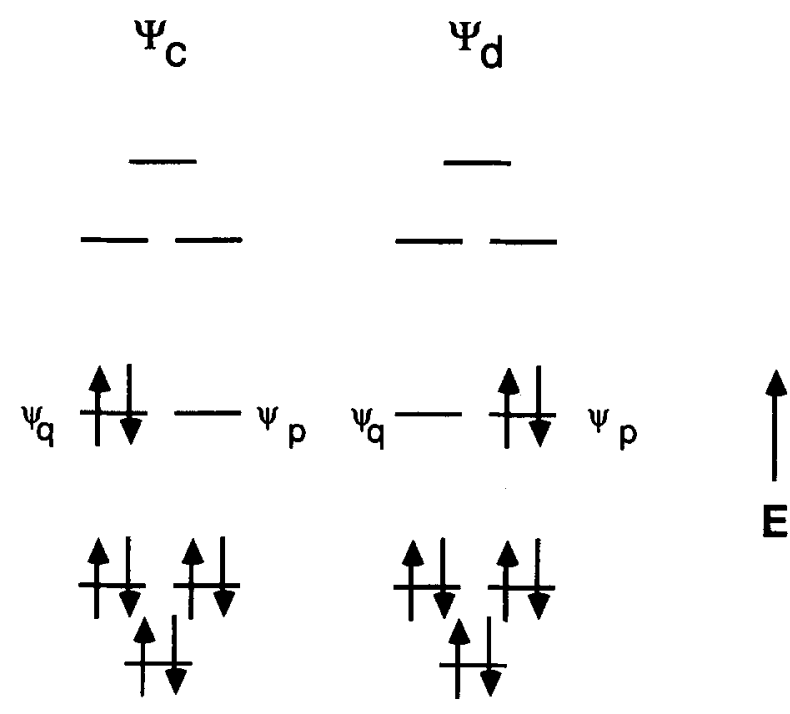

FIG. 9. $\Psi_{c}$ and $\Psi_{d} . \Psi_{c}$ and $\Psi_{d}$ are both eigenfunctions of the Hückel Hamiltonian for cyclooctatetraene. We show in this figure the respective molecular and orbital occupations of $\Psi_{c}$ and $\Psi_{d}$. They differ in their occupation of the nonbonding molecular orbital. $\Phi_{q}=\Phi_{a}+i \Phi_{b}$ while $\Phi_{p}=\Phi_{a}-i \Phi_{b}$, where $\Phi_{a}$ and $\Phi_{b}$ are shown in 25 and 26.

\section{APPENDIX A}

In order to prove the main theorem we need several preliminary results.

As an illustrative example of these preliminary results we consider 27 which has as symmetry elements the permutations shown

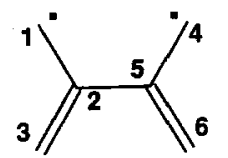

in Table V. We see in this example two results which are generally true and which we state without proof.

\section{Lemma 1}

All $g$ which are in $G$ are either of the: First kind-permutations which interchange starred atoms and starred atoms and/or unstarred atoms and unstarred atoms. Second kind-permutations which interchange unstarred atoms and starred atoms.

\section{Lemma 2}

For connected systems all permutations of the second kind interchange all starred atoms and unstarred atoms. In

TABLE V. Symmetry elements of 27.

Permutations of the first kind:
\[ \begin{array}{r}\text { (13) } \\ \text { (46) } \\ \text { (13) (46) } \\ \text { Permutations of the second kind: } \\ \text { (16) (34) (25) } \\ (14)(36)(25) \\ (1436)(25) \\ (6341)(25)\end{array} \]

the system 27, for example, permutation (14) is not a possible symmetry element. With this as background we now consider $\Psi_{\mathrm{HD}}$. From the variational principle we know that

$$
\left\langle\mid 1 \overline{2} 3 \overline{4} 5 \overline{6} \ldots \| \Psi_{\mathrm{HD}}\right\rangle \equiv \alpha \neq 0
$$

and furthermore that

$$
\begin{aligned}
& \left\langle\mid \overline{1} 2 \overline{3} 4 \overline{5} 6 \ldots \| \Psi_{\mathrm{HD}}\right\rangle \\
& \quad= \pm \alpha\left\{\begin{array}{l}
+ \text { for } 4 N \text { atom systems } \\
- \text { for } 4 N+2 \text { atom systems }
\end{array}\right.
\end{aligned}
$$

From Lemmas 1 and 2 we also see that

$$
\psi_{ \pm} \equiv|1 \overline{2} 3 \overline{4} 5 \overline{6} \ldots| \pm|\overline{1} 2 \overline{3} 4 \overline{5} 6 \ldots|
$$

forms an irreducible representation (ir) of $G . \psi_{+}$and $\psi_{-}$are the same type of ir as $\Psi_{H D}$ itself for, respectively, $4 N$ and $4 N+2$ atom systems ( $\Psi_{\mathrm{HD}}$ is known to be nondegenerate) ${ }^{8}$

Consider now an element $g$ of $G$. Assume $g$ to be an odd permutation of the first kind. Then,

$$
g \psi_{ \pm}=-\psi_{ \pm} \text {. }
$$

In order to prove that $\psi_{ \pm}$and hence $\Psi_{\mathrm{HD}}$ are the most symmetric ir's of $G$ we must prove that all permutations of the first kind are even for all connected systems with a nondegenerate $\Psi_{\text {Hückel }}$.

\section{Lemma 3}

The existence of an odd permutation of the first kind ensures the existence of an orbital such that $H_{\text {Hückel }} \Phi$ $=0 \Phi=0$.

\section{Proof}

In Table VI we list the symmetry elements found in the point groups. We also show in Table VI all the $n$-cycles which are compatible with a given symmetry element. With this as background we can proceed with the proof.

Assume there is an element $g$ of $G$ which is of the first kind and is odd. This implies we have an odd number of $2 n$ -

TABLE VI. Cycle structure of point group elements.

\begin{tabular}{ll}
\hline \hline $\begin{array}{l}\text { Symmetry } \\
\text { element }\end{array}$ & $\begin{array}{l}\text { Possible } n \text {-cycles } \\
\text { for the symmetry element }\end{array}$ \\
\hline$C_{2}$ & 2-cycle, 1-cycle \\
$C_{3}$ & 3-cycle, 1-cycle \\
$C_{4}$ & 4-cycle, 1-cycle \\
$C_{5}$ & 5-cycle, 1-cycle \\
$C_{6}$ & 6-cycle, 1-cycle \\
$C_{7}$ & 7-cycle, 1-cycle \\
$C_{8}$ & 8-cycle, 1-cycle \\
$S_{2}=i$ & 2-cycle, 1-cycle \\
$S_{3}$ & 6-cycle, 3-cycle, 2-cycle, 1-cycle \\
$S_{4}$ & 4-cycle, 2-cycle, 1-cycle \\
$S_{5}$ & 10-cycle, 5-cycle, 2-cycle, 1-cycle \\
$S_{6}$ & 6-cycle, 2-cycle, 1-cycle \\
$S_{7}$ & 14-cycle, 7-cycle, 2-cycle, 1-cycle \\
$S_{8}$ & 8-cycle, 2-cycle, 1-cycle \\
$S_{10}$ & 10-cycle, 2-cycle, 1-cycle \\
$\sigma$ & 2-cycle, 1-cycle
\end{tabular}

We do not list higher rotation elements than eightfold rotations. Nevertheless the conclusions given in the text are valid for higher rotation elements as well. 
cycles as $(2 n+1)$-cycles are even. Let us, as an example, choose $g$ to be

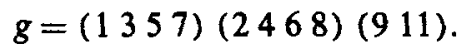

Consider the group $F$ which is the group generated by $g$. There are three functions which belong to the same irreducible representation of $F$ :

$$
\begin{aligned}
& f_{1}=\phi_{1}-\phi_{3}+\phi_{5}-\phi_{7}, \\
& f_{2}=\phi_{2}-\phi_{4}+\phi_{6}-\phi_{8}, \\
& f_{3}=\phi_{9}-\phi_{11} .
\end{aligned}
$$

These are the only three functions which belong to this ir. There are an odd number of them as $g$ was assumed odd. By the pairing theorem ${ }^{10}$ we therefore have one zero eigenvalued eigenvector. Q.E.D.

When we turn to permutations of the second kind we find that for $g_{o}$ (an odd permutation) and $g_{e}$ (an even permutation)

$$
\begin{aligned}
& g_{o} \psi_{ \pm}=\mp \psi_{ \pm}, \\
& g_{e} \psi_{ \pm}= \pm \psi_{ \pm} .
\end{aligned}
$$

In $4 N$ atom systems, we showed earlier that $\psi_{+}$formed the same type of ir as $\Psi_{\mathrm{HD}}$ while for $4 N+2$ atom system it was $\psi_{-}$that did so. We therefore need to prove:

\section{Lemma 4}

For $4 N$ atom systems the existence of an odd permutation of the second kind in $G$ ensures the existence of a zero eigenvalued eigenvector of the one electron $H_{\text {Hückel }}$. For $4 N+2$ atom systems even permutations of the second kind ensure zero eigenvalued eigenvectors.

\section{Proof}

Consider first the $4 N$-atom systems. Assume there is an odd permutation $g$ of the second kind. We observe that $g$ can not contain any odd cycles as this would lead to an interchange of starred with starred atoms. By Lemma $2, g$ contains no 1-cycles. Furthermore $g$ cannot be a $C_{4 n+2}, S_{2 n+1}$ or $S_{4 n+2}$ symmetry element. From Table VI it may be seen that all $C_{4 n+2}, S_{2 n+1}$, and $S_{4 n+2}$ permutations must be even. Similarly we may exclude $\sigma$. (In all these latter cases in order to have $4 \mathrm{~N}$-atoms we need to have an even number of $2 n$-cycles.) The remaining elements are of the type $C_{4 n}$ and $S_{4 n}$.

As an illustrative example consider $S_{4}$. For a $4 N$ system (as Table VI shows) in order for $g$ to be odd these must be an odd number of 4 cycles. We could, for example, have a $g$ :

$g=(1234)$ (5678) (91011 12) (1314) (1516).

Again we consider the group $F$ which is the group generated by $g$. There are three functions which belong to the same irreducible representation

$$
\begin{aligned}
& f_{1}=\phi_{1}+i \phi_{2}-\phi_{3}-i \phi_{4}, \\
& f_{2}=\phi_{5}+i \phi_{6}-\phi_{7}-i \phi_{8}, \\
& f_{3}=\phi_{9}+i \phi_{10}-\phi_{11}-i \phi_{12} .
\end{aligned}
$$

Together these functions produce a block of the Hückel Hamiltonian which is purely imaginary. As we show in Lemma 5 this leads to a pairing theorem applicable to the imaginary block. Hence as in Lemma 3 we have proven the existence of a zero eigenvalued eigenvector. This proves Lemma 4 for $4 N$ atom systems. An identical argument proves the Lemma for $4 N+2$ atom systems. Q.E.D.

\section{Lemma 5}

If $H$ is a Hamiltonian matrix consisting only of purely imaginary matrix elements then if $H t=\lambda t$ (where $t$ is an eigenvector and $\lambda$ its eigenvalue) there exists a $t^{\prime}$, such that $H t^{\prime}=-\lambda t^{\prime}$.

\section{Proof}

$$
\begin{aligned}
& \text { Let } \\
& t=\sum_{j=1}^{N}\left(w_{j}+i v_{j}\right) y_{j},
\end{aligned}
$$

where $w_{j}$ and $v_{j}$ are all real numbers and $\left\{y_{j}\right\}$ form a vector basis set.

Thus by assumption:

$$
\begin{aligned}
\sum_{k=1}^{N} H_{j k} w_{k} & =i \lambda v_{j}, \\
\sum_{k=1}^{N} i H_{j k} v_{k} & =\lambda w_{j} .
\end{aligned}
$$

Now consider

$$
t^{\prime}=\sum_{j=1}^{N}\left[-w_{j}+i v_{j}\right] y_{j} .
$$

Then

$$
H t^{\prime}=-\lambda t^{\prime} \text {. Q.E.D. }
$$

We can now prove the main theorem.

\section{Proof of main theorem}

In the case of $\Psi_{\text {Hückel }}$, we know as there is no degeneracy in the $\Psi_{\text {Hückel }}$ ground state that $\Psi_{\text {Hückel }}$ must belong to the most symmetric representation of $G . \Psi_{\mathrm{HD}}$ is also nondegenerate. We have found a nonzero portion of $\Psi_{\mathrm{HD}}, \psi_{ \pm}$which belongs to the most symmetric representation of $G$. Q.E.D.

\section{APPENDIX B}

For large systems it is difficult to directly calculate the $U / \beta$ value at which the Hubbard transition occurs. We nevertheless can acquire some insight by examining the Hückel and HD asymptotic solutions to the Hubbard model. Two parameters are easily calculated. They are $\Delta E_{\text {Hückel }}$ and $\Delta E_{\mathrm{HD}}$ as shown in Fig. 7. A large $\Delta E_{\text {Hückel }}$ will place the Hubbard crossing at a higher $U / \beta$ value while a large $\Delta E_{\mathrm{HD}}$ will drive the crossing to a lower value. In Table VII we list $\Delta E_{\mathrm{Hückel}}$ and $\Delta E_{\mathrm{HD}}$ for several systems, which undergo a Hubbard transition. It may be seen that the abovementioned effects are indeed observed. For the range of values shown ( $\Delta E_{\text {Hückel }}$ varies from 0.5 to $1.0 \beta$ and $\Delta E_{\mathrm{HD}}$ from 0.5 to 2.0 $J)$ the relation (B1) appears to be approximately correct:

$$
\frac{U_{\text {transition }}}{\beta}=\frac{1}{\Delta E_{\mathrm{HD}}}+2 \cdot \Delta E_{\text {Hückel }} \text {. }
$$


TABLE VII. $U / \beta$ value at Hubbard transition.

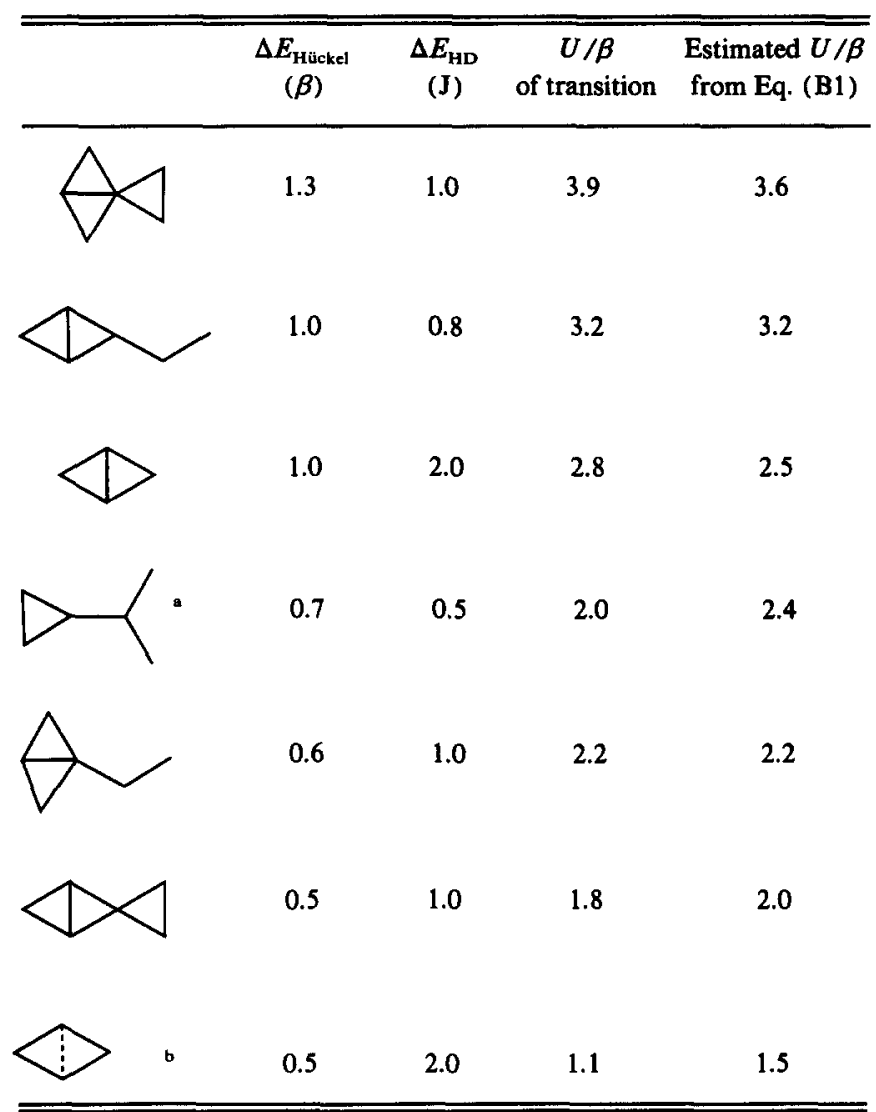

a This system changes from a singlet to a triplet. All other systems in this table undergo singlet to singlet transitions.

${ }^{b}$ Dotted line is a bond at half the normal bond strength.

This relation can clearly not be applied to $\Delta E_{\mathrm{HD}}$ or $\Delta E_{\text {Hückel }}$ very different from the values given above.

\section{APPENDIX C}

Craig $^{12}$ proposed some rules which predict whether an unsaturated hydrocarbon would have a valence bond wave function which belongs to the most symmetrical irreducible representation. In Craig's method, the carbon atoms are labeled with up or down spin. For a given symmetry operation, one determines $p$ and $q$, where $p$ is the number of interchanges between carbon atoms and $q$ is the number of interchanges of spin labels required to restore the original labeling scheme. Craig's rule states that when $p+q$ is even, the ground state belongs to the highest irreducible representation; when $p+q$ is odd, it does not belong to that representation.

It has been noted some years ago ${ }^{17}$ that, for certain molecules (such as 28-30), Craig's rules lead to ambiguous results.
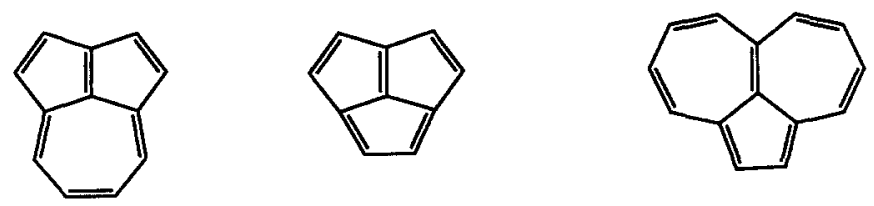

28

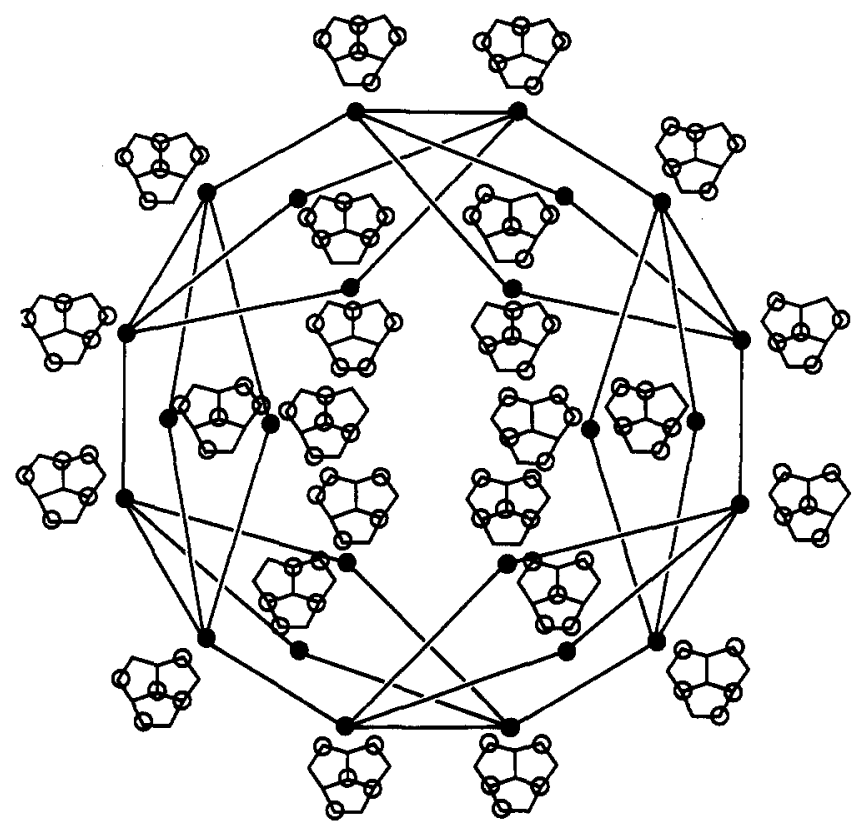

FIG. 10. The LELSDs of 29 are shown on the outer perimeter of the twelvesided polygon shown above. Dark filled circles represent LSDs and long lines show off-diagonal $\Psi_{\mathrm{HD}}$ matrix elements. Near each LSD (dark circles) is a drawing of 29 which indicates the spin alignment of the LSD. Upspin $\pi$ electrons are indicated with an open circle. Down-spin $\pi$ electrons are without circles. In the interior of the 12-sided polygon, the twelve LSDs which are not allowed in $A_{2}$ symmetry are shown. Each of these twelve LSDs is connected in $H_{\mathrm{HD}}$ to two LELSDs.

Thus, in the case of 28 , two possible labeling schemes are shown in 31 and 32. For 31, $p+q$ is even while for 32, $p+q$ is odd.

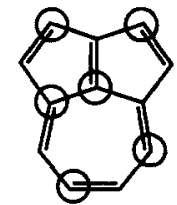

31

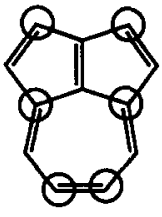

32
This ambiguity in Craig's rule can be resolved by using methods similar to those outlined in the text. We consider, as a simple example, the system 29. There are 12 LELSDs whose off-diagonal matrix elements are shown pictorially in Fig. 10. If one assumes a $C_{3 v}$ symmetry, the interaction of these 12 LELSDs leads one to predict a ${ }^{3} A_{2}$ ground state. Direct calculation of $H_{\mathrm{HD}}$ shows the ground state is of ${ }^{1} E$ symmetry. The reason for this difference is that there are twelve LSDs which interact closely with the LELSDs, which are forced to have a zero coefficient under ${ }^{3} A_{2}$ symmetry. We show these LSDs and their relation to the LELSDs in Fig. 10 . Inclusion of these twelve LSDs leads one to a ${ }^{1} E$ ground state. The analysis is therefore clear. The molecule 29 has a degenerate $\Psi_{\mathrm{HD}}$ and we therefore expect the molecule to distort so as to lose its threefold symmetry axis. try (Macmillan, New York, 1982). 
${ }^{2}$ Recent papers which show some of the current applications of one electron theory are (a) G. J. Miller, J. K. Burdett, C. Schwarz, and A. Simon, Inorg. Chem. 25, 4437 (1986); (b) W. Tremel, R. Hoffmann, and J. Silvestre, J. Am. Chem. Soc. 108, 5174 (1986). Important earlier papers include: (c) M. H. Whangbo, R. Hoffmann, and R. B. Woodward, Proc. R. Soc. London Ser. A 366, 23 (1979); (d) R. Hoffmann, J. Chem. Phys. 39, 1397 (1963); (e) R. B. Woodward and R. Hoffmann, The Conservation of Orbital Symmetry (Verlag Chemie, Weinheim, 1970).

${ }^{3}$ Important papers on the HD spin Hamiltonian are (a) D. Mattis, Phys. Rev. 130, 76 (1963); (b) E. Lieb and D. Mattis, J. Math Phys. 3, 749 (1962); (c) E. Lieb, T. Schultz, and D. Mattis, Ann. Phys. 16, 407 (1961); (d) D. J. Klein, J. Chem. Phys. 77, 3098, (1982); (e) S. A. Alexander and D. J. Klein, J. Am. Chem. Soc. 110, 3401 (1988).

${ }^{4}$ E. Z. Hückel, Phys. 70, 204 (1932); (b) A. Streitweiser, Molecular Orbitals theory for Organic Chemists (Wiley, New York, 1961).

${ }^{5}$ See the discussion in L. Salem, The Molecular Orbital Theory of Conjugates Systems (Benjamin, New York, 1966).

${ }^{6}$ R. B. Woodward and R. Hoffmann, The Conservation of Orbital Symmetry (Verlag Chimie, Weinheim, Germany, 1970).

${ }^{7}$ (a) P. Du, D. A. Hrovat, T. W. Borden, P. M. Lahti, A. R. Rossi, and J. A. Berson, J. Am. Chem. Soc. 108, 5072 (1986); (b) P. M. Lahti, A. R. Rossi, and J. A. Berson, ibid. 107, 2273 (1985); (c) J. L. Goodman and J. A. Berson ibid. 107, 5409 (1985); (d) D. E. Seeger and J. A. Berson, ibid. 107, 5409 (1985); (d) D. E. Seeger and J. A. Berson, ibid. 105, 5146 (1983); (e) W. T. Borden and E. R. Davidson, ibid. 99, 4587 (1977); (f) But note the exceptional system found in P. Dowd, W. Chang, and Y. H. Paik, J. Am. Chem. Soc. 109, 5284 (1987).

${ }^{8}$ (a) E. H. Lieb and F. Y. Wu, Phys. Rev. Lett. 20, 1445 (1986); (b) J. E. Hirsch, Phys. Rev. B 31, 4403; (c) D. J. Klein, Pure Appl. Chem. 55, 299 (1983); (d) D. J. Klein, C. Nelin, S. Alexander, and F. A. Matsen, J.
Chem. Phys. 77, 3101 (1982); (e) S. Lee, J. Chem. Phys. 90, 2732 (1989). '(a) J. Hubbard, Proc. R. Soc. London Ser. A 276, 238 (1963); (b) H. Shiba and P. Pincus, Phys. Rev. B 5, 1966 (1972).

${ }^{10}$ C. A. Coulson and H. C. Longuet-Higgins, Proc. R. Soc. London Ser. A 192, $16(1948)$.

"Y. S. Lee, K. F. Freed, H. Sun, and D. L. Yeager, J. Chem. Phys. 79, 3862 (1983).

${ }^{12}$ (a) This result was first noted in D. P. Craig and A. Maccoll, J. Chem. Soc. 1949, 965; (b) D. P. Craig, ibid. 1951, 3175; (c) A. Julg and P. Francois, Theor. Chim. Acta 11, 128 (1968); (d) the initial formulation of the problem by D. P. Craig leads to Craig's rules. See the discussion in Appen$\operatorname{dix} \mathbf{C}$.

${ }^{13}$ There are two different types of Hubbard transitions. The transition can be a transition from a singlet state to a singlet state. This is the case for pentalene. There may also be a transition from a singlet to a higher spin multiplet. In this section, we verify that there is a crossing within the sing let manifold. Such a crossing ensures the existence of a Hubbard transition. It is quite possible, though, that at a $U / \beta$ value, which is smaller than the singlet-singlet crossing a singlet-triplet crossing in the ground state has occurred. This is the case for the system shown in Fig. 4(b) and in 15.

${ }^{14}$ (a) B. Kitschke and H. J. Lindner, Tetrahedron Lett. 29, 2511 (1977); (b) Angew. Chem. Int. Ed. Engl. 15, 106 (1976); (c) H. J. Lindner and J. Stegemann, Tetrahedron Lett. 29, 2515 (1977).

${ }^{15} \mathrm{H}$. C. Longuet-Higgins and L. Salem, Proc. Soc. London Ser. A 251, 172 (1959).

${ }^{16}$ H. J. Dauben, J. D. Wilson, and J. L. Laitz, in Nonbenzenoid Aromatics, edited by J. P. Snyder (Academic, New York, 1971), Vol. II. See also Ref. 5, p. 205.

${ }^{17}$ R. A. Abramovitch and K. L. McEwen, Can. J. Chem. 43, 2616 (1965). 